\title{
SIMBORGS AND SIMULATED TASK ENVIRONMENTS FOR ENGINEERING NEXT GENERATION WORKSTATIONS FOR INTELLIGENCE ANALYSTS
}

\author{
Wayne D. Gray, Michael J. Schoelles, \& V. Daniel Veksler \\ Rensselaer Polytechnic Institute \\ [grayw/schoem/vekslv]@rpi.edu
}

\begin{abstract}
Intelligence analysts require innovative software environments for searching massive databases, hypothesis generation and test, record keeping, crosschecking and so on. Yet, as important as such tools are, their development cannot be allowed to ignore the hard won lessons from cognitive science and humancomputer interaction - that human cognition is embodied cognition and that interface design has a large role to play in increasing productivity and reducing human error. We propose an approach to the cognitive engineering of integrated task environments by the use of simulated cyborgs (simBorgs) that combine computational embodied-cognitive models of interactive behavior with artificial intelligence based reasoning components in a simulated task environment. Our goal is the creation of intelligent agents, simBorgs, that will work tirelessly to perform usability testing on various combinations of tasks and interfaces.
\end{abstract}

For a simulated human user to be useful in testing interface designs how much of human behavior must be simulated in a cognitively plausible manner? For those interested in interface design, the problem is that task knowledge and even cognitive processes (Ericsson \& Kintsch, 1995) are very task-specific. Do we need a cognitively plausible model of an expert architect to evaluate the interface of an architectural CAD-CAM system? More to the current point, how much of an Intelligence Analyst's (IA) expertise do we need to model to evaluate interfaces used in the IA's task environment?

An equally important question concerns the nature of the task environment required for usability testing. Although it might seem ideal to test interface designs in the context of the actual work environment, the complexity of such environments may make it difficult or impossible to determine which interface features contribute to what aspects of human performance. As the nature of the work changes from, for example, the routine interactions required by telephone operators (Gray, John, \& Atwood, 1993) to the never-the-same-twice problems faced by IAs the difficulties in relating design to performance also increase.

Fortunately, the answer to both of these problems is the same; namely, to build a complex, but limited simulated task environment in which a limited number of domain-specific scenarios can be played out. In effect, create a scaled world (Gray, 2002) that exercises a representative range of the skills and talents required for on-the-job performance.

Interface problems are often considered a nuisance, but not a major factor in task performance of motivated, highly trained experts. As we explain in the next section, this nuisance perspective is wrong. Interface problems cannot be overcome by motivation and training, and they are inevitably compounded in complex task environments. Our solutions to cognitively engineering task environments are discussed in sections two and three. Section two introduces the Sage World task environment for IAs. Section three discusses the simBorg concept.

All of the current work is directed towards improving the software task environment of the IA. However, we believe the approach is quite general and can be adapted for other applications.

\section{More Than a Nuisance: Why Interface Problems Defy Motivation and Training to Negatively Affect Performance and Errors}

In this section we provide a very general discussion of why interface problems cannot be simply overcome and why they are an all but inevitable concomitant to complex task environments. In Resolving the Paradox of the Active User we discuss empirical and theoretical reasons for believing that in many situations even the most motivated of users will not reach optimal performance due to interface issues. In Profound Embodiment we discuss our reasons for arguing that as an agent's task environment increases in complexity the number of different ways in which an agent can and will implement the same subtask increases. Soft Constraints Theory provides a short discussion of why some compositions are preferred to other compositions even when they lead to 
suboptimal performance in certain tasks.

\section{Resolving the Paradox of the Active User}

The "paradox of the active user" (Carroll \& Rosson, 1987) is the persistent use of inefficient procedures in interactive environments by experienced or even expert users when demonstrably more efficient procedures exist.

$\mathrm{Fu}$ and Gray (in press) identified regularities across the inefficient procedures used by three very different categories of users in three very different sorts of applications. For each data set, we first satisfied ourselves that the preferred procedures used by some users were indeed less efficient than the recommended procedures. We then amassed evidence, for each set, and concluded that when a preferred procedure was used instead of a more efficient, recommended procedure, the preferred procedure tended to have two major characteristics: (1) the preferred procedure was a well-practiced, generic procedure that was applicable either within the same task environment in different contexts or across different task environments, and (2) the preferred procedure was composed of interactive components that brought fast, incremental feedback on the external problem states.

The support amassed for these characteristics leads to a new understanding of the paradox. In interactive tasks, people are biased towards the use of general interactive procedures that effect immediate change. This bias can persist for years and may result in stable, if suboptimal, performance. If innovative applications are to achieve their full potential, the challenge for researchers is to understand the basis of the paradox so that interfaces can be cognitively engineered to allow users to achieve the full potential of the application.

\section{Profound Embodiment}

"The embodied view is that the environment and perceiver should not be separated but should be viewed as an integrated system" (Ballard, 2002). Properties of functional cognition and of the perceived world are in a tight loop. Properties of our cognitive, perceptual, and action systems determine how much and what aspects of the world we perceive; whereas properties of the world determine the mixture of cognitive, perceptual, and action operations required for us to perceive it.

Cognitive resources may be in-the-head or in-theworld. The exact mix of resources used is not fixed, but variable depending on the exact mix of conditions provided by the environment and the cognitive controller. This view is similar to Clark's notion of Profound Embodiment where the boundary between agent and world is "rather permeable and reconfigurable" (Clark, 2004).

The Profound Embodiment perspective has interesting implications for human-computer interaction. The number of potential compositions available to an agent is a partial function of the number of different objects (and different ways of interacting with those objects) in the agent's task environment. As new and innovative software systems are added to the agent's task environment, the agent's pattern of use of any one system should diverge more and more from developer defined expectations of optimal use.

\section{Soft Constraints Theory}

Soft Constraints Theory applies the rational, leasteffort framework (Anderson, 1990; Payne, Bettman, \& Johnson, 1993; Simon, 1956) to the nondeliberate choices that occur at the embodiment level. The composition of activities chosen is neither uniquely specified by the task environment nor determined by the agent. Rather, when selection is non-deliberate or automatic the combination with the best cost-benefit tradeoff (i.e., expected value) is chosen.

Calculations of expected value take into account an agent's past successes and failures as well as their past experience with the cost of applying procedures in different task environments. Hence, the three components in making a selection are the environment, the processing resources of the agent, and the agent's past history.

Soft constraints imply that the cognitive controller may recruit different sets of activities to accomplish the same microstrategy or unit task. The origin of a resource, in-the-world or in-the-head (Gray \& Fu, 2004), in the software package currently used or from another one in the task environment $(\mathrm{Fu} \&$ Gray, in press), is not important. What is important is the agent's prior history of successes and costs of using that resource.

Soft constraints do not guarantee an optimal solution for any given interactive behavior but, given enough experience, may result in a stable, if suboptimal, solution across the complete task environment (Fu \& Gray, 2004).

\section{Summary}

The theory of soft constraints can be applied to predict performance in a given task environment. Used for this purpose, it should be possible to predict patterns of stable, suboptimal tradeoffs that occur with different task environments as well as 
how changes to one component (e.g., one software package) or the addition of a new component (e.g., a new software package) will affect overall performance.

\section{The Sage World Simulated Task Environment}

Sage is intended to be a scaled world (Ehret, Gray, \& Kirschenbaum, 2000; Gray, 2002) that focuses on a subset of the functional relationships found in the IA's task environment. Like all scaled worlds (Gray, 2002), Sage seeks to preserve the functional relationships of research interest while paring away others.

Key to the Sage scaled world are a series of scenarios developed by and for the intelligence community. Although diverse and heterogeneous, one thing that IAs have in common is their love of historical intelligence case studies and their enjoyment of working through intelligence scenarios developed as training exercises or as intellectual puzzles.

Sage will incorporate these scenarios into what can be considered a sophisticated role playing game for the experienced IA. Indeed, to some degree, Sage will resemble the best of today's role-playing games; a sophisticated blend of WarCraft ${ }^{\mathrm{TM}}$, Civilization ${ }^{\mathrm{TM}}$, and SimCity ${ }^{\mathrm{TM}}$. The end result will be a scaled world in which the quest given to the IA will be similar to the tasks given the same agent in daily life. As with many role-playing games, time and money are limited and both are required to acquire information, as well as to develop and test hypotheses. Hence, the IA's goal is to derive the best solution possible by a wise use of time and money. The main differences between Sage and the IA's real world are that the pace will be faster, the penalty for failure much lower, and the same scenario can be played with a wide variety of new tools and techniques. Of not incidental importance, everything about Sage can be instrumented and studied so as to better understand the IA's cognitive processes and how the nature of the task and the nature of the tools available for accomplishing the task influence those processes.

\section{simBorgs in Sage World}

Cyborgs are science-fiction creations that are part human and part machine. We see the creation of simulated cyborgs as the solution to building models that interact with a variety of software tools in a manner that requires domain expertise. Like the scifi cyborgs, our simBorgs combine human and machine components. Our simBorgs consist of high- fidelity models of human interactive behavior, knowledge schemas derived from expert humans as they perform realistic tasks, and a black-box module.

The power of the simBorg approach comes from the constraints inherent in the Sage scaled world. Although the Sage World scenarios will be realistic, they will be limited in number and will be designed to play out over a 2-4 hr period. Hence, Sage World scenarios contrast greatly with real world scenarios where the same exact problem never recurs and even small problems can take days to resolve. Another key difference between the world and Sage World is that Sage World scenarios are always solvable and always have known solutions.

Even with these strong constraints, constructing high-fidelity computational cognitive models of IA problem solving is a long-term research project. Fortunately, more immediate solutions are available.

If one is not interested in cognitive fidelity, it is often simpler to model human performance by a mathematical equation, statistical techniques such as multiple regression, or a machine learning algorithm than it is to build a high-fidelity computational cognitive model. For example, Deep Blue beat Gary Kasparov at Chess. However, Deep Blue was not a model of human chess playing expertise. Designing an AI system that will beat the world's best human at chess has been done. Designing a computational cognitive model that will play world-class chess in the same manner as the best human plays chess is more difficult and has not been done.

As Sage World scenarios are fixed and limited we can completely describe possible solution paths. Hence, rather than modeling human problem solving for these solution paths, we can rely on various black-box techniques, thereby focusing our efforts on predicting how subtle differences in costs and benefits of interactive methods affect performance and errors. As discussed below, a three-tier architecture emerges from this focus.

\section{Tier A - Interactive behavior}

The interactive behavior tier interacts directly with the task environment much in the manner that people interact with it. This is the level of highest cognitive fidelity and represents an off-the-shelf use of ACT-R 5.0 (Anderson, Bothell, Byrne, \& Lebiere, 2002). For this tier, the composition of activities needed to implement a particular subtask follows from soft constraints evaluations based on the environment, the processing resources of the agent, and the agent's past history.

Like the humans, the Interactive Behavior Module will interact differently with different scenarios in 
the same task environment. It will also interact differently when the same scenario is repeated in different task environments (i.e., different configurations of tools). Having a family of cognitively realistic models of interactive behavior will enable us to quickly predict the influence that various designed environments have on human performance.

\section{Tier B - Knowledge Schemas}

Success at designing better task environments requires more than models that interact with their environment the way that people interact. The models' activities must be directed towards the same information acquisition goals as people and the information collected must be used for reasoning, problem-solving, or hypothesis generation and testing much as for humans.

To finesse this problem, tier B consists of knowledge schemas that guide interactive behavior using sets of scenario-specific schemas. These schemas are derived by knowledge engineering techniques (primarily protocol analysis) from human experts as they solve a given scenario. The schemas will guide information acquisition activities and cache the results. (Developing these scenariospecific schemas is, in itself, a major knowledge engineering effort.) As used here, the schema framework has its origins in the Project Nemo work (Gray, 2003).

Schemas may be considered the knowledge structures that Ericsson and Kintsch (1995) refer to as long-term working memory. There may well be multiple schemas for the same task. Indeed, as we elaborate below, it is probably more accurate to think of sets of schemas rather than one schema per formal method. Different sets of schemas may enable the use of different black-box methods. Schema variations within a set may feed the same black-box method different combinations of information or information that varies in its reliability and validity.

\section{Tier C - The Black Box}

Tier $\mathrm{C}$ is the black-box module. This module may contain a variety of methods for problem solving or reasoning in the task domain. Depending on the problem, the method chosen may be a machinereasoning algorithm, Bayesian network, multiple regression model, or algebra equation that simply computes an answer. (Indeed, we can envision writing simple arithmetic Lisp functions that compute different decision-making algorithms; i.e., compensatory versus non-compensatory ones, etc.)
The essence is that the black-box method provides an answer that a human might give, but the process by which the answer is derived bears no resemblance to human information processing.

Selecting one among several black-box methods will be based on considerations of expected value. As such, the expected value for a method will accumulate as a function of the costs of acquiring information for that method and the benefit of choosing that method as opposed to alternative methods. Hence, although the process by which a black-box method works will be independent of considerations of human limits, the process by which a method is selected will be part and parcel of those limitations.

Such soft constraints applied to the selection of black-box methods may result in alternative methods becoming eligible to fire after different types and amounts of data have been gathered. For example, rather than having three methods that compete at the same time, it may be the case that Method 1 is enabled with less or different data than the other two methods. In this case Method 2 might require all of the data collected by Method 1 plus more, whereas Method 3 might require different data. In this way, the method that first becomes eligible to fire is subject to the successes, costs, performance, and errors of tier A interactive behavior.

Unlike human decision-makers, the black-box methods will always work perfectly. The caveat is GIGO (garbage-in garbage-out). If the first two tiers feed the black-box method old information or wrong information, the black-box method will accurately compute the best answer that can be obtained from the information provided. Likewise, if the interface makes it simple and easy to collect some types of information, but difficult to collect other types then, in our example, Method 3 might be selected even though Method 2 yields a better or more complete answer.

The simBorg approach promises to provide a general way of exploring how differences in interface design lead people to tradeoff effectiveness for efficiency in the strategies they use. The goal is to be able to separate the low-level information acquisition and interaction processes from the higher-level, problem-specific expertise required to do a particular job.

\section{From Cognitive Science to Cognitive Engineering}

The idea of building simBorgs is a bold and challenging extension of the current state of the art 
in computational cognitive modeling. simBorgs represent a marriage of black-box approaches with a high-fidelity model of embodied cognition capable of interacting with a variety of interface designs and making the same least-effort tradeoffs as human agents.

The simBorg approach is made possible by the presence of a scaled world (Sage World) that mimics key features of the IA's task environment. This combination of cognitive modeling with black-box algorithms in a scaled world enables the creation of intelligent agents, simBorgs, that will work tirelessly to perform usability testing on various combinations of tasks and interfaces proposed for IAs.

In the longer term, as our work proceeds, simBorgs provide a vehicle for incrementally replacing black-box approaches with high-fidelity cognitive models of the problem solving performed by human IA's. Hence for the Sage World project, the ultimate fate of the simBorgs is to be replaced by high-fidelity computational cognitive models of IAs. This will enable us to approach the ultimate goal of cognitive engineering; namely, to tailor the designed environment to best fit the ways in which the human agent thinks, perceives, and acts.

\section{Acknowledgements}

Support for the writing of this paper was provided in part by a subcontract to Rensselaer Polytechnic Institute from contract \#MDA-904-03-C-0408 to Booz Allen Hamilton from the Advanced Research and Development Activity (ARDA). Support for the development of the simBorg concept and Soft Constraints Theory have also come from AFOSR \#F49620-03-1-0143 and ONR \#N000140310046.

\section{References}

Anderson, J. R. (1990). The adaptive character of thought. Hillsdale, NJ: Lawrence Erlbaum Associates.

Anderson, J. R., Bothell, D., Byrne, M. D., \& Lebiere, C. (2002). An integrated theory of the mind. Retrieved October 17, 2002, from http://actr.psy.cmu.edu/papers/403/IntegratedTheory.pdf

Ballard, D. H. (2002). Our perception of the world has to be an illusion. Journal of Consciousness
Studies, 9(5-6), 54-71.

Carroll, J. M., \& Rosson, M. B. (1987). Paradox of the active user. In J. M. Carroll (Ed.), Interfacing thought: Cognitive aspects of human-computer interaction. Cambridge, MA: MIT Press.

Clark, A. (2004). Embodiment and ecological control. Retrieved April 9, 2004, from http://www.cognitivesciencesociety.org/colloquiu m/archive.html

Ehret, B. D., Gray, W. D., \& Kirschenbaum, S. S. (2000). Contending with complexity: Developing and using a scaled world in applied cognitive research. Human Factors, 42(1), 8-23.

Ericsson, K. A., \& Kintsch, W. (1995). Long-term working memory. Psychological Review, 102(2), 211-245.

Fu, W.-T., \& Gray, W. D. (2004). Suboptimal tradeoffs in information seeking. submitted for publication.

Fu, W.-T., \& Gray, W. D. (in press). Resolving the paradox of the active user: Stable suboptimal performance in interactive tasks. Cognitive Science.

Gray, W. D. (2002). Simulated task environments: The role of high-fidelity simulations, scaled worlds, synthetic environments, and microworlds in basic and applied cognitive research. Cognitive Science Quarterly, 2(2), 205-227.

Gray, W. D. (2003). Final Report for Project Nemo: Subgoaling and subschemas for submariners. Troy, NY: Rensselaer Polytechnic Institute.

Gray, W. D., \& Fu, W.-T. (2004). Soft constraints in interactive behavior: The case of ignoring perfect knowledge in-the-world for imperfect knowledge in-the-head. Cognitive Science, 28(3), 359-382.

Gray, W. D., John, B. E., \& Atwood, M. E. (1993). Project Ernestine: Validating a GOMS analysis for predicting and explaining real-world performance. Human-Computer Interaction, 8(3), 237-309.

Payne, J. W., Bettman, J. R., \& Johnson, E. J. (1993). The adaptive decision maker. New York: Cambridge University Press.

Simon, H. A. (1956). Rational choice and the structure of the environment. Psychological Review, 63, 129-138. 